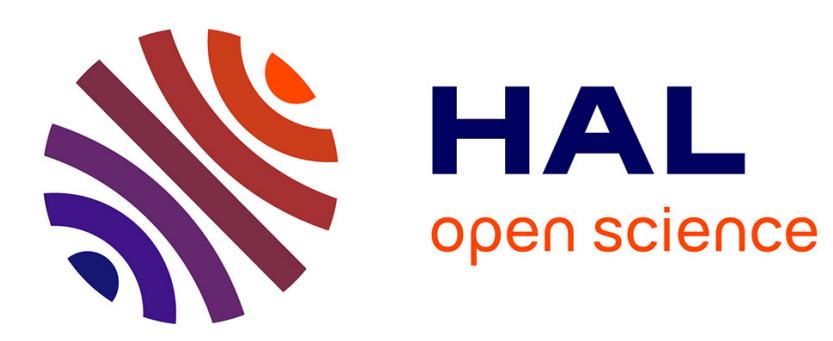

\title{
La colonisation en image dans les documentaires pour enfants : médiation éditoriale et écriture de l'Histoire
} Julia Bonaccorsi

\section{To cite this version:}

Julia Bonaccorsi. La colonisation en image dans les documentaires pour enfants: médiation éditoriale et écriture de l'Histoire. Spirale - Revue de Recherches en Éducation , 2007, 40 (1), pp.27 - 36. 10.3406/spira.2007.1391 . halshs-01635254

\section{HAL Id: halshs-01635254 \\ https://shs.hal.science/halshs-01635254}

Submitted on 23 May 2018

HAL is a multi-disciplinary open access archive for the deposit and dissemination of scientific research documents, whether they are published or not. The documents may come from teaching and research institutions in France or abroad, or from public or private research centers.
L'archive ouverte pluridisciplinaire HAL, est destinée au dépôt et à la diffusion de documents scientifiques de niveau recherche, publiés ou non, émanant des établissements d'enseignement et de recherche français ou étrangers, des laboratoires publics ou privés. 


\section{La colonisation en image dans les documentaires pour enfants :} médiation éditoriale et écriture de l'Histoire

Julia Bonaccorsi

\section{Citer ce document / Cite this document :}

Bonaccorsi Julia. La colonisation en image dans les documentaires pour enfants : médiation éditoriale et écriture de l'Histoire. In: Spirale. Revue de recherches en éducation, $n^{\circ} 40,2007$. Images pour apprendre. pp. 27-36;

doi : https://doi.org/10.3406/spira.2007.1391

https://www.persee.fr/doc/spira_0994-3722_2007_num_40_1_1391

Fichier pdf généré le 31/03/2018 


\title{
Résumé
}

Les ouvrages documentaires pour enfant constituent un lieu d'enseignement de l'Histoire, nonformel et péri-scolaire. L'iconographie y est importante et variée selon des registres divers allant du dessin fictionnel à la photographie d'archive. L'analyse sémiotique d'un corpus de documentaires historiques sur la colonisation met en évidence la complexité du rôle dévolu à l'image comme vecteur d'apprentissage, lié ici à des logiques éditoriales, génériques, les spécificités d'une discipline scientifique et de ses traditions, mais aussi son rôle social qui articule transmission du savoir et éducation citoyenne. Les choix iconographiques sont révélateurs des rapports à la source historique, à l'événement, au fait et à l'Histoire, transmis par les auteurs/ éditeurs. L'énonciation éditoriale qui met en scène les images dans ces documentaires attribue au lecteur des rôles interprétatifs induits par les projets éditoriaux des collections mais aussi par les modèles d'écriture de l'Histoire et des registres iconographiques qu'elle mobilise. C'est l'identité même du jeune lecteur qui est en jeu : spectateur, historien ou héritier.

\begin{abstract}
Documentary books for children - with their rich iconography, varying from archival photographs to fictional drawings - are used as non-formal tools for the teaching of history. In this semiotical study we analyze a corpus of historic documentaries. We show how the iconography is related to editorial, gender, and disciplinary factors, and how the iconographic choices reveal relationships with historical sources and with historical events. Editorial enunciation assigns interpretative roles to the reader which derive from editorial projects, historical interpretative models and iconographical registers. The young reader is successively spectator, historian and inheritor.
\end{abstract}




\title{
LA COLONISATION EN IMAGE DANS LES DOCUMENTAIRES POUR ENFANTS : MÉDIATION ÉDITORIALE ET ÉCRITURE DE L'HISTOIRE
}

\begin{abstract}
Résumé : Les ouvrages documentaires pour enfant constituent un lieu d'enseignement de l'Histoire, non-formel et péri-scolaire. L'iconographie y est importante et variée selon des registres divers allant du dessin fictionnel à la photographie d'archive. L'analyse sémiotique d'un corpus de documentaires historiques sur la colonisation met en évidence la complexité du rôle dévolu à l'image comme vecteur d'apprentissage, lié ici à des logiques éditoriales, génériques, les spécificités d'une discipline scientifique et de ses traditions, mais aussi son rôle social qui articule transmission du savoir et éducation citoyenne. Les choix iconographiques sont révélateurs des rapports à la source historique, à l'événement, au fait et à l'Histoire, transmis par les auteurs/éditeurs. L'énonciation éditoriale qui met en scène les images dans ces documentaires attribue au lecteur des rôles interprétatifs induits par les projets éditoriaux des collections mais aussi par les modèles d'écriture de l'Histoire et des registres iconographiques qu'elle mobilise. C'est l'identité même du jeune lecteur qui est en jeu : spectateur, historien ou héritier.
\end{abstract}

Mots-clés : Education non-formelle - Littérature de jeunesse - Ouvrage documentaire - Iconographie - Enonciation éditoriale - Vulgarisation scientifique - Histoire.

Le 25 septembre 2006, le Journal télévisé de France 2 annonçait une possible revalorisation des pensions de guerre des soldats des anciennes colonies, information corrélée à la sortie du film Indigènes ${ }^{l}$. L'accompagnement médiatique et pédagogique de ce film cristallise la vivacité du débat sur la mémoire coloniale qui, depuis 2005, a largement ouvert le questionnement centré sur la guerre d'Algérie dans les années quatre-vingt-dix aux processus de colonisation et de décolonisation ${ }^{2}$.

Dans ce contexte relevant non seulement d'enjeux de mémoire mais aussi d'enjeux politiques contemporains, la question de la transmission pédagogique de l'histoire est centrale ; par exemple, les manuels scolaires font l'objet d'analyses qui

\footnotetext{
${ }^{1}$ Un film de Rachid Bouchareb, septembre 2006.

${ }^{2}$ Pour une synthèse de cette chronologie et des publications qui y sont liées, voir : Bancel et Blanchard (2006).
} 


\section{J. BONACCORSI}

semblent y chercher la clef de paradoxes mémoriaux ou encore le signe (le lieu du crime) d'une idéologie univoque. La loi du 23 février 2005 préconisant l'évocation $\mathrm{du}$ «rôle positif de la présence française » dans le cadre de la colonisation renforce ce statut dévolu au manuel scolaire comme lieu où s'élabore le récit national. Est pointé en particulier le rôle de l'iconographie, renforcement et ancrage des stéréotypes pour les uns, ouverture par l'activité d'analyse pour les autres (Gaulupeau, 2005). À cet égard, l'histoire éditoriale et pédagogique des manuels est éclairante, puisqu'elle pointe l'usage croissant de l'iconographie dans les manuels à partir des années quatre-vingt, servant «à refroidir les questions chaudes», en rejetant dans l'analyse de documents la prise en charge des points de vue (Lantheaume, 2006). L'image devient le lieu médiateur du débat.

Les ouvrages documentaires pour la jeunesse constituent un autre lieu d'enseignement de l'histoire, non-formel et péri-scolaire ${ }^{3}$. L'iconographie y est importante et variée selon des registres divers allant du dessin fictionnel à la photographie d'archive. Comme le souligne Daniel Jacobi, l'image dans les documentaires pour la jeunesse est aussi le lieu où s'affirment les points de vue et les partis pris, qu'ils soient directement liés à la médiation scientifique ou au projet éditorial dans une perspective plus large (Jacobi, $2005:$ 109).

Nous intéresser à l'image dans un corpus thématique de documentaires pour la jeunesse permet de traverser les partis pris en nous penchant sur la matérialité même des images mobilisées ; leur statut et leur régime ; les modalités de la médiation scientifique qui s'opère. Ainsi, nous ferons l'analyse des images de documentaires ayant trait à la question de la colonisation, dans un contexte polémique qui traite, en particulier, de la place occupée par l'iconographie historique dans les manuels scolaires.

Le premier volet de cet article porte sur les catégories d'images mobilisées dans ces documentaires pour la jeunesse en montrant ce qu'elles révèlent des projets éditoriaux, mais aussi des registres d'écriture de l'Histoire. Nous pointons alors comment l'iconographie de la question coloniale dans ces ouvrages propose des « scènes » archétypales. Enfin, nous montrons comment les modalités de médiation repérées s'articulent avec la dialectique mémoire/Histoire qui traverse les débats sur la question coloniale.

\section{DU BON USAGE D'UN CORPUS THÉMATISÉ}

La littérature documentaire, y compris les encyclopédies pour la jeunesse, se différencie du reste de la production par la nature de son projet de vulgarisation et de médiation des savoirs. Ce genre éditorial complexe croise les registres informatif, explicatif, ou narratif pour répondre à son projet d'éducation non-formelle et de vulgarisation scientifique. La production éditoriale contemporaine de documentaires est organisée autour de trois grands «genres » : la leçon du manuel scolaire, le récit

\footnotetext{
${ }^{3}$ Cette catégorisation est avant tout structurelle : dans les pratiques, les documentaires servent fréquemment de support pédagogique.
} 
fictionnel et l'encyclopédie, modèles classiques du texte de vulgarisation (Bonaccorsi et Jacobi, 2005).

Quelle imagerie renvoie à la colonisation dans les documentaires pour la jeunesse ? Selon quelles catégories éditoriales ces images sont-elles mobilisées ?

Le corpus sur lequel porte la recherche (12 documentaires de 1990 à 2005) a été sélectionné à partir d'une centaine d'ouvrages depuis $1870^{4}$. Un retour bref sur la constitution de ce corpus soulève certains éléments significatifs pour notre analyse : colonisation ne constituait pas un mot-clef pertinent, de même que décolonisation. Nous avons donc procédé à plusieurs décadrages après un premier feuilletage de documentaires en élargissant aux thèmes suivants : histoire de France, pays (Algérie, Afrique, Maghreb), thématiques liées (intégration, immigration, esclavage). Nous emploierons dans cet article le mot colonisation pour recouvrir ce qui relève de la question coloniale, de la conquête coloniale à la période post-coloniale.

L'analyse du corpus fait apparaître deux catégories principales du genre : le manuel (la leçon d'histoire) et le récit fictionnel qui fait jouer, selon différents plans, les frontières entre réalité scientifique et imaginaire.

Nous faisons l'hypothèse que le thème colonisation va déterminer des registres spécifiques de médiation historique par l'image, marqués à la fois par les habitus de la discipline et de la pédagogie de celle-ci, et par les problématiques épistémologiques et historiographiques liées, en particulier, à la dialectique mémoire/Histoire (Nora, 1997). Sandrine Lemaire montre ainsi combien il est nécessaire de rapprocher dans l'enseignement Histoire nationale et Histoire coloniale, nécessité à la fois disciplinaire et exigence sociétale liée, en particulier, à l'articulation de la question coloniale et de l'immigration (Lemaire 2006 : 62). Les débats contextuels constituent un ensemble de paradigmes qui traversent la production éditoriale de documentaires sur la question coloniale et participent des décisions éditoriales et auctoriales $^{5}$.

Ainsi, l'usage des images est lié à des pratiques éditoriales et au projet des collections. C'est aussi le lieu où s'affirme un exercice de l'Histoire, comme discipline scientifique : l'énonciation éditoriale met en scène les matériaux de cette discipline comme le témoignage, les archives et ses pratiques d'écriture (de Certeau, 1975).

\section{DES CLICHÉS DU COLONIAL À UNE POLYPHONIE DE LA MÉMOIRE}

Figements : motifs de l'iconographie et scènes du genre

La diversité des formes éditoriales et des registres iconographiques qui frappe au premier regard est atténuée par une lecture plus approfondie des images. En

\footnotetext{
${ }^{4}$ Les ouvrages sélectionnés s'adressent à un public adolescent. Ses bornes temporelles (de 1990 à aujourd'hui) permettent de travailler en synchronie.

${ }^{5}$ Création des collections J'ai vécu (Bayard), Histoire d'Histoire (Rue du Monde), Citoyens en herbe (Gallimard jeunesse)...
} 


\section{J. BONACCORSI}

effet, la répétition des motifs des illustrations attire alors l'attention, et conduit à une synthèse de la question coloniale en 4 ou 5 motifs.

Hélène Puiseux montre comment dès la Monarchie de Juillet, cette question fait l'objet d'une imagerie figée, des tableaux d'Horace Vernet aux gravures de l'Illustration, puis aux images d'actualité. «Pour nous qui le voyons à la lumière des suites de la colonisation, le plus frappant, dans ce matériel finement gravé, est qu'il offre la même thématique et les mêmes éléments humains et géographiques que ceux livrés par les actualités filmées, des décennies plus tard, jusque pendant la guerre d'Algérie. (...). L'image est constituée et fixée dès les années 1860 et restera figée dans son discours même lorsque le mouvement cinématographique interviendra.» (Puiseux, 1997 : 105)

Dans les documentaires de notre corpus, les motifs des images sont relatifs à une chronologie de la question coloniale.

La conquête coloniale est rarement représentée. Quand elle l'est, c'est par le biais d'images d'Epinal et de tableaux d'Horace Vernet, ainsi que par les cartes (carte des explorations qui synthétisent une histoire coloniale longue: L'Afrique, Terres et peuples) ${ }^{6}$. C'est le thème de l'esclavage qui symbolise ces premiers temps du colonialisme : gravures de ventes d'esclaves, modèle réduit du négrier Brookes.

L'école est le lieu par excellence de la transmission des connaissances: l'énonciation éditoriale croise alors l'école du lecteur et celle des colonies soit par figuration de l'école contemporaine (en toile de fond dans L'intégration, représentée par le dessin dans Midi pile, soit par contraste (L'Algérie).

La continuité ou la circulation de ces motifs quelles que soient les spécificités matérielles des images est remarquable.

La colonisation en Afrique ou en Indochine est peu représentée. Quand elle l'est, c'est par l'esclavage et sa violence (Terres et peuples) ; par le casque du colonisateur symbolisant l'administration; par le travail forcé dans des photographies peu lisibles où les corps des hommes se mêlent à la matière; enfin, par les troupes coloniales (tirailleurs sénégalais et annamites, au combat dans L'intégration, ou prenant la pose dans La France)

À l'inverse, la vie quotidienne en Algérie fait l'objet d'une iconographie riche mais très homogène (rues de la ville, cafés et salons de thé des colons ${ }^{7}$ ). Femmes voilées et djellabas sont mêlées aux chapeaux melons et robes fifties : tout concourt à mettre en scène le cosmopolitisme et l'inter-culturalité parfois même sur le mode stéréotypé de l'exotisme, signifié en particulier par le recours à l'aquarelle (J'étais enfant, Midi pile. Réalistes, ces dessins s'inspirent souvent des croquis des carnets de voyage. L'illustration dessinée est étroitement associée au récit fictionnel, mais elle est ancrée dans la réalité, servant davantage à installer les personnages et

\footnotetext{
${ }^{6}$ Le lecteur trouvera en fin d'article la description complète des ouvrages cités dont le nom est abrégé dans le corps du texte.

${ }^{7}$ On retrouve le Victoria bar dans une photographie de Midi pile et en dessin dans Alger. Cette circulation photographie/dessin relève non seulement d'une pratique professionnelle de l'illustration qui s'appuie sur de la documentation, mais elle renvoie aussi à la place de l'interprétation dans le projet de transmission du savoir (Gervais, 2003 ; Lehni, 2006).
} 
le récit dans une réalité historique, qu'à réinterpréter l'événement et à le renvoyer vers l'imaginaire. Dans les Carnets d'Orient, l'archive est mêlée au dessin fictionnel (dessin des Une des journaux ou encore gravure représentant Abd El Kader dans Rue de la Bombe).

La conquête de l'indépendance est souvent omise et reléguée au traitement de la guerre d'Algérie. L'Afrique depuis 1945 consacre un chapitre à la marche vers l'indépendance de l'Afrique en convoquant une iconographie variée (portraits de chefs nationalistes, statue de colonialiste renversée, violence des répressions en Afrique du Sud, barricades à Alger (Alger) qui illustre, symboliquement ou techniquement, le texte long et exhaustif dans lequel la conquête de l'indépendance algérienne n'est qu'un exemple. L'analyse comparée des documentaires révèle une récurrence des motifs de la guerre relatifs à la guerre d'Algérie. Ainsi, les plans photographiés ou dessinés de patrouilles dans la montagne blanche et aride ou dans la casbah d'Alger décrivent une guerre d'occupation et de retour à l'ordre marquée par des violences intenses et brèves (attentats, représailles). L'issue en est figurée par la foule en liesse dans les rues d'Alger (Midi pile, Alger, J'ai vécu) mais aussi par l'image du départ des pieds-noirs, de dos, sur le pont d'un bateau (La France, J'ai vécu, J'étais enfant, Alger).

Dans Alger, l'enfant narrateur est le témoin naiff d'une guerre qui le dépasse et qui met fin à son enfance ainsi qu'à une culture décrite de manière assez angélique. Aux événements historiques est entrelacée une énonciation nostalgique qui désigne non pas un regret de l'Algérie française, mais une intimité mémorielle : nostalgie d'une enfance insouciante et, par là, nostalgie d'une Algérie révolue ${ }^{8}$ ?

Cet exemple est caractéristique d'une médiation qui passe par la mémoire (ou des mémoires) avant d'être une médiation de l'Histoire. Dans J'ai vécu, l'usage des photographies provenant de fonds privés associe les registres du récit individuel et collectif : elles représentent le narrateur en soldat, mais sont aussi des synecdoques du soldat dans la guerre. Les photographies de papiers d'identités servent l'authenticité du témoignage ou du récit fictionnel (J'ai vécu, La guerre fantôme) et retracent une histoire politique vers la décolonisation et l'indépendance (J'ai vécu, L'Algérie).

L'énonciation de ces mémoires est réalisée selon les modes stéréotypés de la figuration d'objets (billes, illustrés dans Alger, une Peugeot noire, les valises dans J'étais enfant, de la subjectivité de l'aquarelle et des couleurs, de motifs figés. Il convient alors de nous intéresser à la prise en charge interprétative des images et au rôle dévolu au lecteur en regard de l'Autre.

Mémoire/Histoire : quel regard sur l'Autre?

Quelles peuvent donc être les modalités d'une médiation de l'Histoire qui passe par une iconographie à la fois très hétérogène mais traversée par des paradigmes récurrents au niveau thématique et formel ? Quatre modalités de médiation par l'image se dégagent de notre analyse et renvoient à différents niveaux de la dialecti-

${ }^{8}$ En cela, la conclusion de l'ouvrage n'est pas moins ambiguë : « La liberté avait un prix. » 


\section{J. BONACCORSI}

que mémoire/Histoire : européo-centrée, prenant en charge l'altérité, vecteur du témoignage, englobante dans une histoire commune.

La première modalité est qualifiée d'européo-centrée en ce qu'elle attribue au lecteur un point de vue extérieur, mais situé en Europe. Ainsi, le texte d'accompagnement d'une photographie représentant la construction d'une voie ferrée ( $L a$ France) illustre bien cette modalité : "Nombreux sont les "nègres" qui meurent à la tâche.» (p. 11). La mise entre guillemets du mot «nègre » suppose de la part du lecteur un travail d'interprétation basé sur des compétences sémantiques qui relèvent, d'une part, d'une connaissance d'une histoire culturelle de la colonisation, et, d'autre part, d'une position externe.

Mais cette modalité n'est pas nécessairement négative, et elle concerne plutôt des genres éditoriaux comme le manuel ou l'encyclopédie qui reposent davantage sur une énonciation informative.

La prise en charge de l'altérité, seconde modalité, se manifeste diversement. Par exemple, le co-texte précisera les conditions de production des images et analysera leur réception, comme dans L'Afrique : XVIII... : l'image d'Epinal de la bataille de Médine est ainsi commentée «les images d'Epinal familiarisent les Français avec la conquête coloniale. » La photographie de l'Almami Samori Touré est comparée à un dessin du $\mathrm{XIX}^{\mathrm{e}}$ présenté sur la page précédente : «cette photographie le montre digne mais résigné à l'exil (...). Elle contraste avec la représentation imaginaire de l'Almami (p. 28) qui orientalise les symboles du pouvoir.» (p. 31).

La médiation est réalisée selon deux plans : présenter l'Autre en tant qu'acteur de la question coloniale, et expliciter le regard colonial sur l'Autre, qui passe, justement, par une iconographie imaginaire et orientalisée qui fausse la vérité historique.

Le choix éditorial de Terres et peuples met en scène dans l'espace scriptovisuel des objets (costumes, outils, statuettes, etc.) qui s'apparentent à une collecte ethnologique. C'est par l'objet que la question coloniale est traitée, qu'il s'agisse d'objets colonialistes et esclavagistes mais aussi de représentations indigènes. Ainsi, les reproductions photographiques détourées de trois statuettes attirent l'attention, «représentant des colons allemands». L'une d'entre elles «est plus grande que les autres, sans doute pour montrer l'importance du personnage». Le documentaire donne à voir une interprétation de la question coloniale par l'Autre, invitant le jeune lecteur européen à se l'approprier, mais en conservant cependant une distance ethnologique que le modalisateur sans doute souligne.

Ainsi, cette modalité de la médiation analyse le discours sur l'Autre en même temps qu'il rend présent cet Autre par un discours et des représentations qui lui appartiennent. Mais cette prise en charge de l'altérité reste ténue et non affirmée dans le projet éditorial.

La part accordée à la voix de l'Autre peut prendre une forme revendiquée dans l'intitulé même des collections par l'usage, en particulier, de la première personne (J'ai vécu, J'étais enfant). Cette troisième modalité de médiation s'appuie sur le genre du témoignage, fiction ou récit réel et peut-être univoque, ou polyphonique. Dans tous les cas, il s'agit de mettre en scène des mémoires singulières. Les illustra- 
tions de l'ouvrage de Leïla Sebbar, J'étais enfant, articulent différents registres narratifs qui correspondent aux dialogues et témoignages que la narratrice retranscrit dans son journal, heure par heure, avant le départ pour la France. Les aquarelles donnent à voir une mosaïque de souvenirs : des mémoires particulières de l'Histoire, mémoires pied-noir ou arabe, comme de petites touches du vécu. L'enfant narrateur fait entrer le lecteur dans une intimité émotionnelle de l'événement historique.

Le témoignage sert aussi une rhétorique du portrait archétypal, comme dans J'ai vécu où les photographies sont légendées par chacun des narrateurs (militaire français, fellagha, harki, pied-noir). Le témoignage se construit avec le récit d'une mémoire individuelle que l'énonciation éditoriale institue en archétype, et, en creux, le récit d'une histoire individuelle qui se reconstruit à partir de l'archive privée. L'avant-propos introduit ainsi ce double récit «Ce livre donne la parole à des hommes et des femmes qui ont vécu l'Histoire plus qu'ils ne l'ont faite. » Le principe du récit biographique archétypal repose sur le postulat que les histoires individuelles sont cohérentes et constituent des trajectoires logiques et déterminées. L'interprétation des images est déléguée à chacun des narrateurs. La superposition des regards et des voix sur le même événement implique que le lecteur reste neutre, historien.

Enfin, la quatrième modalité de médiation repose aussi sur le registre du souvenir mais l'intègre dans un archi-discours qui fait du lecteur un acteur à part entière. Ainsi, dans Midi pile, les choix narratifs et l'énonciation éditoriale convergent pour proposer une lecture polyphonique. Séparée du texte principal et de l'illustration fictionnelle, une photographie d'archive (qualifiée de «documents d'épo$q u e$ ») illustre un texte distinct du récit fictionnel par sa forme : en caractères plus petits et en italique. Les photos sont colorisées de manière uniforme, ce qui apporte une grande lisibilité : chaque texte peut être lu de manière indépendante, et le lecteur n'a pas à statuer sur les images, fiction ou archive ${ }^{9}$. Le triple récit (les enfants contemporains qui assistent au rendez-vous, les adultes témoins de la guerre et l'Histoire événementielle) est exemplaire d'une médiation à tiroirs qui prend en charge l'Histoire collective et les mémoires individuelles mais les réinscrit dans une histoire commune (celle des enfants de la cité qui sert de contexte).

De même, L'intégration se réfère à la question coloniale dans le cadre narratif très précis du genre épistolaire à partir duquel les enfants échangent commentaires et informations. Cet énoncé est alors enrichi par des photographies de troupes au repos et de tirailleurs sénégalais, images associées au dessin de la jeune narratrice visitant un monument aux morts avec son grand-père. La présence de l'affiche de l'Exposition coloniale de 1931 contribue à la rhétorique visuelle d'une histoire commune. Dans ce documentaire, cette rhétorique doit servir avant tout l'intégration républicaine.

Ces deux exemples montrent comment la lecture et l'interprétation de l'iconographie est encadrée par un archi-récit qui détermine les régimes sémiotiques des

${ }^{9}$ On renverra pour l'analyse du double récit de vulgarisation à (Jacobi, 2003). 


\title{
J. BONACCORSI
}

images en définissant le rôle du jeune lecteur dans la narration elle-même, non pas comme spectateur, témoin ou historien mais comme héritier.

Au terme de cette analyse, nous souhaiterions mettre en relief deux éléments. Nous avons montré comment l'énonciation éditoriale qui met en scène les images dans ces documentaires propose des modes de lecture et attribue au lecteur des rôles interprétatifs induits par les projets éditoriaux des collections mais aussi par les modèles d'écriture de l'Histoire et des registres iconographiques qu'elle mobilise. Les choix iconographiques sont révélateurs des rapports à la source historique, à l'événement, au fait et à l'Histoire, transmis par les auteurs/éditeurs. Enfin, c'est l'identité même du jeune lecteur qui est en jeu : spectateur, historien ou héritier.

Ainsi, l'analyse sémiotique de ces documentaires met en évidence la complexité du rôle dévolu à l'image comme vecteur d'apprentissage, lié ici à des logiques éditoriales et génériques, une discipline scientifique et ses traditions ainsi que son rôle social qui articule transmission du savoir et éducation citoyenne. Or, les documentaires pour la jeunesse en associant image et texte selon des relations plus ou moins lisibles et explicitées, jouant des registres d'inventivité graphique et de créativité narrative rendent parfois complexe le positionnement du jeune lecteur qui doit statuer et interpréter des images sans être forcément accompagné (comme c'est le cas pour les manuels scolaires). À ce titre, la triple distinction graphique et narrative que propose Midi pile est exemplaire : le documentaire réunit deux époques et trois récits, en distinguant deux registres iconographiques, l'illustration fictionnelle et la photographie d'archive. Les places attribuées au témoin, à l'historien et au jeune citoyen y sont clairement définies. L'ouvrage documentaire remplit alors un triple rôle : enseigner l'Histoire, transmettre une pratique historiographique, et, enfin, proposer au lecteur une place dans la mémoire collective.

Julia BONACCORSI

Université Paris XII - Val-de-Marne

Laboratoire Céditec EA 3119

\begin{abstract}
Documentary books for children - with their rich iconography, varying from archival photographs to fictional drawings - are used as non-formal tools for the teaching of history. In this semiotical study we analyze a corpus of historic documentaries. We show how the iconography is related to editorial, gender, and disciplinary factors, and how the iconographic choices reveal relationships with historical sources and with historical events. Editorial enunciation assigns interpretative roles to the reader which derive from editorial projects, historical interpretative models and iconographical registers. The young reader is successively spectator, historian and inheritor.

Keywords : Non-formal education - Documentary book - Iconography - Editorial enunciation - Scientific translation - History.
\end{abstract}




\section{Bibliographie}

Bancel N. \& Blanchard P. (2006) « Mémoire coloniale : résistances à l'émergence d'un débat» - Autrement : Culture post-coloniale 1961-2006 126, (22-41).

Barthes R. (1972) «Les planches de l'Encyclopédie » - in : Nouveaux essais critiques. Paris : Seuil.

Bernard P. \& Rollot C. (2005) «Les points d'interrogation des manuels » - Le Monde, 25 décembre.

Bonaccorsi J. \& Jacobi D. (2005) «De l'Atelier du Père Castor aux Yeux de la découverte : créativité éditoriale et médiation des savoirs dans l'édition du livre documentaire jeunesse » - in : Legendre, Bertrand, Robin, Christian (dir.) Figures de l'éditeur (109-122). Paris : Nouveau Monde Éditions.

De Certeau M. (1975) L'écriture de l'histoire. Paris : Gallimard, Folio Histoire.

Ferro M. (1983) « Histoire vue d'Europe, Histoire vue d'ailleurs » - La Revue des Livres pour Enfants 92-93 (43-46).

Gaulupeau Y. (2005) «Les manuels par l'image : la colonisation » - Histoire de l'Éducation 58 (103-135).

Gervais T. (2003) « Premiers usages de la photographie dans le journal l'Illustration »-Études Photographiques 13 (157-161).

Jacobi D. (2005) Les sciences communiquées aux enfants. Grenoble : PUG.

Jacobi D. (2003) «Un livre documentaire scientifique pour enfants : entre vulgarisation et littérature »-Questions de Communication 4.

Lantheaume F. (2006) «Les difficultés de la transmission scolaire : le lien AlgérieFrance dans les programmes d'histoire, les manuels et l'enseignement en France » - Colloque Pour une histoire critique et citoyenne, ENS LSH.

Lemaire S. (2006) « Histoire nationale et histoire coloniale : deux histoires parallèles »-Autrement Culture post-coloniale 1961-2006 (53-68).

Lehni C. (2006) «De l'exploration au récit grand public»-Études Photographiques 18 (73-95).

Le Van Ho M. (1988) «Le documentaire historique pour enfants en France de 1970 à 1987 : la Révolution française » - La Revue des Livres pour Enfants 122-123 (57-68).

Nora P. (1997) «Entre Mémoire et Histoire» — in : Les lieux de mémoire (23-42). Paris : Gallimard (Quarto).

Puiseux H. (1997) Les figures de la guerre. Paris : Gallimard. 


\section{J. BONACCORSI}

\section{Corpus analysé}

\begin{tabular}{|c|c|c|c|c|c|}
\hline Date & Auteur & Titre & Edition & Collection & $\begin{array}{l}\text { Renvoi abrégé } \\
\text { dans le texte }\end{array}$ \\
\hline 1990 & $\begin{array}{l}\text { S. Baynham, F. } \\
\text { Carlier; ill. : } \\
\text { Camel Pictures }\end{array}$ & $\begin{array}{l}\text { L'Afrique depuis } \\
1945\end{array}$ & $\begin{array}{l}\text { Gamma } \\
\text { Trécarré }\end{array}$ & $\begin{array}{l}\text { Conflits du } \\
\text { XXe siècle }\end{array}$ & $\begin{array}{l}\text { L'Afrique depuis } \\
1945\end{array}$ \\
\hline 1995 & $\begin{array}{l}\text { Y. Ayo ; Ill. } \\
\text { photographies : } \\
\text { R. Moller, G. } \\
\text { Dann et D. Gar- } \\
\text { ner }\end{array}$ & Terres et peuples & $\begin{array}{l}\text { Gallimard jeu- } \\
\text { nesse }\end{array}$ & $\begin{array}{l}\text { Les Yeux de la } \\
\text { découverte }\end{array}$ & Terres et peuples \\
\hline 1996 & $\begin{array}{l}\text { I. Ibazebo ; } \\
\text { Trad.: T. Guidi- } \\
\text { celli }\end{array}$ & L'Afrique & Bayard-Okapi & J'explore & L'Afrique \\
\hline 1997 & $\begin{array}{l}\text { L. Sebbar ; Ill. : } \\
\text { C. Belkadi }\end{array}$ & $\begin{array}{l}\text { J'étais enfant en } \\
\text { Algérie }\end{array}$ & $\begin{array}{l}\text { Editions du } \\
\text { Sorbier }\end{array}$ & J'étais enfant & J'étais enfant \\
\hline 1999 & $\begin{array}{l}\text { C. Braconnier ; } \\
\text { Ill. : S. Bataille }\end{array}$ & L’intégration & $\begin{array}{l}\text { Gallimard jeu- } \\
\text { nesse }\end{array}$ & $\begin{array}{l}\text { Citoyens en } \\
\text { herbe }\end{array}$ & L'intégration \\
\hline 2000 & K. Delobbe & $\begin{array}{l}\text { La France au } \\
\text { XXe siècle }\end{array}$ & PEMF & $\begin{array}{l}\text { Bonjour } \\
\text { l'histoire }\end{array}$ & La France \\
\hline 2001 & $\begin{array}{l}\text { J.-P. Vittori ; } \\
\text { Ill. : J. Ferrandez }\end{array}$ & Midi pile & Rue du Monde & $\begin{array}{l}\text { Histoire } \\
\text { d'Histoire }\end{array}$ & Midi pile \\
\hline 2002 & $\begin{array}{l}\text { Gilles Bonotaux } \\
\text { et Hélène Las- } \\
\text { serre, Ill. Gilles } \\
\text { Bonotaux. }\end{array}$ & $\begin{array}{l}\text { Alger 1954-1962. } \\
\text { Il y avait parfois } \\
\text { des nuages sur } \\
\text { Alger }\end{array}$ & $\begin{array}{l}\text { Autrement jeu- } \\
\text { nesse }\end{array}$ & $\begin{array}{l}\text { Quand ils avaient } \\
\text { mon âge... }\end{array}$ & Alger \\
\hline 2002 & J. Ferrandez & $\begin{array}{l}6 \text { titres dont } L a \\
\text { guerre fantôme } \\
(2002) \text { et } R \text { Re de la } \\
\text { Bombe (2004) }\end{array}$ & Casterman & Carnets d'Orient & Carnets d'Orient \\
\hline 2003 & J.-J. Jordi & $\begin{array}{l}\text { L'Algérie. Des } \\
\text { origines à nos } \\
\text { jours }\end{array}$ & Autrement & Junior/Histoire & L'Algérie \\
\hline 2004 & $\begin{array}{l}\text { A. d'Abbundo ; } \\
\text { Ill. : P. Dolé- } \\
\text { mieux }\end{array}$ & $\begin{array}{l}\text { J'ai vécu la guerre } \\
\text { d'Algérie }\end{array}$ & Bayard jeunesse & Dossiers Okapi & J'ai vécu \\
\hline 2005 & O. Goerg & $\begin{array}{l}\text { L'Afrique : XVIIIe- } \\
\text { XXIe siècle. Du } \\
\text { Sud du Sahara au } \\
\text { Cap de Bonne- } \\
\text { Espérance }\end{array}$ & Autrement & Junior/Histoire & L'Afrique : XVIII \\
\hline
\end{tabular}

